\title{
ROBOTS NADADORES TIPO FLAGELO BACTERIANO DE PEQUEÑAS DIMENSIONES: DESARROLLO DE PROTOTIPOS Y PLATAFORMAS DE PRUEBA
}

\author{
Javier Silva García, Javier Prieto Arranz, Inés Tejado, Emiliano Pérez, Blas M. Vinagre \\ Escuela de Ingenierías Industriales, Universidad de Extremadura, (06006) Badajoz. \\ e-mail: $\{$ jsilvaga, japrietoa\}@alumnos. unex.es, $\{$ itejbal, emilianoph, bvinagre $\} @$ @unex.es
}

\section{Resumen}

Este artículo presenta las primeras experiencias de nuestro equipo de investigación en el desarrollo de robots nadadores tipo flagelo helicoidal de pequeñas dimensiones (de unos pocos centimetros) capaces de navegar en un fluido en condiciones de bajo número de Reynolds. Se presentan dos prototipos de robots que difieren entre sí principalmente en la forma de actuación: robot nadador con actuación magnética externa, cuyo desplazamiento es posible gracias a un imán permanente en rotación, y robot nadador autopropulsado, mediante un motor de pequeñas dimensiones. Se describen tanto los prototipos desarrollados como las plataformas de prueba asociadas, así como los primeros experimentos realizados. El principal objetivo es demostrar que la actuación de los robots funciona, mientras que la propulsión de los mismos es una cuestión más delicada que requiere de una profundización e investigación mayor.

Palabras clave: Robot, nadador, pequeña dimensión, actuación, propulsión, fluido.

\section{INTRODUCCIÓN}

Hay previsiones de que la robótica de pequeña escala, es decir, robots de dimensiones de varios centímetros o incluso inferior (escala micro o nano), revolucione muchos aspectos en diversos ámbitos. También llamada microrrobótica en muchos trabajos de la bibliografía especializada, como aplicaciones más destacables se pueden mencionar las siguientes: en medicina, como técnicas mínimamente invasivas $[8,15]$ o en diágnostico y tratamiento de enfermedades [2, 11, 13], en tareas inspección, por ejemplo de infrastructuras [27, 34] o de maquinaria [3], en labores de búsqueda y rescate en catástrofes $[10,14,31]$, e incluso en entretenimiento y juegos [30]. El lector interesado puede consultar $[16,26]$ y las referencias que allí se incluyen para una revisión más detallada de otras aplicaciones de este tipo de robots.

Sin embargo, trabajar en escala micro impone muchos desafíos, principalmente relativos a la fabrica- ción de los robots, control y suministro de energía. La locomoción también es una cuestión clave a considerar en este tipo de robots, ya que sobre ella también se reflejan los efectos del escalado. En ese sentido, el número de Reynolds (en adelante, $R e$ ), relación entre las fuerzas inerciales y las de fricción o viscosas, dependiendo del medio en el que se encuentre el robot, suele ser otra consideración a la hora del diseño del método de locomoción del robot. En la actualidad, una de las tendencias que inspira los avances en este campo consiste en reproducir las funciones de locomoción de ciertos animales, especialmente el nado de criaturas acuáticas y el vuelo de insectos [28].

En este contexto, el objetivo de este artículo es presentar las primeras experiencias de nuestro equipo de investigación en el desarrollo de robots nadadores de pequeñas dimensiones capaces de navegar en fluidos en condiciones de bajo Re, es decir, aquellos en los que la inercia es tan pequeña que cualquier momento aplicado al fluido se anula casi inmediatamente por las fuerzas viscosas. Como solución de navegación para los robots se ha optado por emular la de las bacterias con flagelos de forma helicoidal utilizando tanto actuación externa como autónoma. Para el primer caso, el prototipo de robot desarrollado navega gracias al campo magnético inducido por un imán en rotación, mientras que el robot autopropulsado lleva un pequeño motor. Asimismo, se describen las plataformas de prueba asociadas a cada caso desarrolladas y los experimentos realizados. Tanto la construcción de los prototipos con actuación externa y autopropulsado como la de los bancos de prueba asociados están basados en los trabajos presentados en [4] y [21], respectivamente.

Cabe mencionar que estos prototipos, debidamente escalados a dimensiones micro o nano con la tecnología de fabricación adecuada, pretenden ser los primeros pasos hacia nuevas soluciones técnicas para procedimientos médicos y quirúrgicos menos invasivos. En concreto, el objetivo final de la línea de investigación iniciada con este trabajo es el desarrollo de microrrobots nadadores tipo flagelo artificial bacteriano capaces de navegar por el sistema circulatorio humano. Por tanto, a los 
desafíos ya impuestos por trabajar en la escala micro habría que sumarles los derivados de la navegación en el cuerpo humano.

El resto del artículo está estructurado como sigue. En la sección 2 se exponen brevemente los fundamentos del movimiento a bajos $R e$ y la forma de obtener estos valores bajos para robots con dimensiones de centímetros. El mecanismo de propulsión de los flagelos artificiales bacterianos se fundamenta en la sección 3 . Las secciones 4 y 5 están dedicadas a la descripción de los prototipos de los robots con actuación externa y autopropulsado. En la sección 6 se muestran los experimentos realizados con los prototipos desarrollados. Finalmente, la sección 7 resume brevemente lo desarrollado y apunta algunas perspectivas futuras de nuestro trabajo a modo de conclusión.

\section{MOVIMIENTO A BAJOS NÚMEROS DE REYNOLDS}

La hidrodinámica de objetos nadadores macroscópicos viene gobernada por la ecuación de Navier-Stokes, que para un fluido incompresible viene dada por la ecuación

$$
\frac{\partial \mathbf{V}}{\partial t}+(\mathbf{V} \cdot \nabla) \mathbf{V}-\frac{\mu}{\rho} \nabla^{2} \mathbf{V}=-\frac{1}{\rho} \nabla P+\mathbf{F},
$$

donde $t$ es el tiempo, $\rho$ es la densidad del fluido, $\mu$ la viscosidad dinámica, $\mathbf{V}$ es el vector velocidad, $P$ es la presión, y $\mathbf{F}$ representa otras fuerzas externas. Dicha ecuación es aplicable a regímenes con alto $R e$, es decir, aquellos en los que los términos inerciales (los dos primeros en la ecuación (1)) son mucho mayores que los viscosos. Todo indica que un nadador en estos regímenes se basa en la inercia para nadar.

Sin embargo, a pequeña escala (hasta cientos de micras), las fuerzas viscosas dominan sobre las inerciales dando como resultado bajos $R e$, experimentando los objetos nadadores una hidrodinámica diferente. En estos regímenes la ecuación (1) se reduce a la ecuación de Stokes:

$$
-\frac{\mu}{\rho} \nabla^{2} \mathbf{V}+\frac{1}{\rho} \nabla P=\mathbf{F} .
$$

Las estrategias de locomoción utilizadas en estos casos son bastante diferentes a las utilizadas a nivel macroscópico, pues la deriva debida a la inercia ya no funciona. La estrategia de la venera (scallop) de cerrar sus valvas a gran velocidad y abrirlas a una velocidad más lenta no produce propulsión en estos dominios, puesto que el cambio de velocidad en los movimientos genera fuerzas inerciales insignificantes. Por lo tanto, para nadar en estos casos es necesario un movimiento no recíproco, irreversible en el tiempo, y los dos que más se han estudiado en la literatura son los que se obtienen batiendo o rotando un flagelo, o lo que es lo mismo, imprimiendo en el flagelo una onda plana o helicoidal.

En el caso de querer obtener a escala macroscópica condiciones de bajos $R e$, se tendrá que escalar el resto de las magnitudes que intervienen en este parámetro adimensional. Teniendo en cuenta la expresión para el número de Reynolds

$$
R e=\frac{\rho V L}{\mu},
$$

establecido el tamaño del robot $(L)$, se deberá utilizar un fluido que, a las velocidades de desplazamiento esperadas $(V)$, tenga la relación $\rho / \mu$ adecuada. $\mathrm{O}$ de manera equivalente, una viscosidad cinemática $(\eta)$ adecuada, ya que $\eta=\mu / \rho$.

\section{PROPULSIÓN EN FLAGELOS ARTIFICIALES BACTERIANOS}

Las principales cuestiones a considerar a la hora del diseño de robots nadadores de pequeñas dimensiones son dos: el método de actuación y el mecanismo de propulsión. Con respecto a la primera, la energía necesaria para el movimiento del robot puede obtenerse utilizando métodos externos o internos, es decir, el robot puede ser actuado de forma remota o autónoma. Para actuación remota, se pueden utilizar varios enfoques. La actuación magnética se puede utilizar para propulsión, control y seguimiento [4, 21, 33]. Otros métodos de actuación remota suponen el uso de ondas sonoras o ultrasónicas, y también pueden utilizarse para seguimiento del robot $[7,12]$. Independientemente del método de actuación, nadar en entornos con bajo Re requiere procedimientos diferentes a los utilizados en gran escala. Con respecto al mecanismo de propulsión, en la escala micro se consideran dos métodos, ambos inspirados en dispositivos biológicos: onda helicoidal y onda plana [1, 5, 9, 18] (véase la Figura 1). Como se ha comentado, este trabajo se centra en el primer tipo. En [29] se presenta el estudio del mecanismo de propulsión de un robot tipo flagelo bacteriano plano.

En el caso que nos ocupa, nos referiremos como flagelo a una hélice rígida con una geometría fija, como se muestra en la Figura 2. Como se puede observar, los parámetros geométricos que definen el flagelo son: el radio del filamento $(a)$, el radio helicoidal $(R)$, el paso de la espiral $(\lambda)$ y su longitud axial $(L)$. El ángulo de paso $(\theta)$ está relacionado con $\lambda$ y $R$ mediante $\theta=\tan ^{-1}(2 \pi R / \lambda)$ 


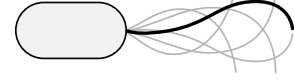

(a) (b)

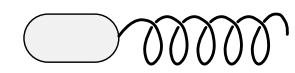

Figura 1: Mecanismos de propulsión de un flagelo artificial bacteriano: (a) Onda viajera (b) Helicoidal

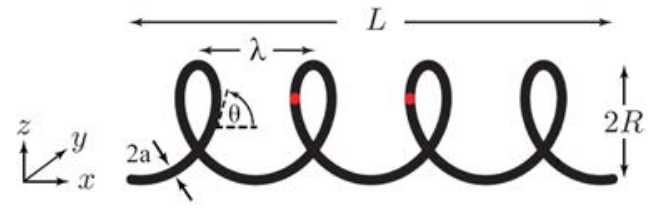

Figura 2: Geometría de un flagelo helicoidal

En condiciones de bajo Re, un flagelo ejerce una fuerza axial $F$ y un momento $T$, relacionados con la velocidad lineal, $V$, y de rotación $\Omega$ del flagelo mediante la ecuación matricial:

$$
\left(\begin{array}{c}
F \\
T
\end{array}\right)=\left(\begin{array}{ll}
A & B \\
B & C
\end{array}\right) \cdot\left(\begin{array}{c}
V \\
\Omega
\end{array}\right),
$$

donde $A=D_{x} / V, B=F_{x} / \Omega$ y $C=T_{x} / \Omega$, siendo $F$ y $T$ la fuerza axial de empuje y el momento de un flagelo que rota pero no se desplaza y $D$ la fuerza axial de arrastre de un flagelo que se desplaza sin rotar. La matriz de $2 \times 2$, conocida en algunos trabajos como propulsiva o resistiva, depende únicamente de la geometría del flagelo.

La teoría de la fuerza resistiva obtiene estas fuerzas y momento del movimiento de un flagelo integrando las fuerzas locales en cada segmento infinitesimal del propio flagelo. Las fuerzas locales se calculan utilizando unos coeficientes de arrastre por unidad de longitud en las direcciones normal y tangencial al segmento, $C_{n}$ y $C_{t}$, respectivamente. Los resultados son:

$$
\begin{array}{r}
F_{x}=-(\Omega R)\left(C_{n}-C_{t}\right) \sin \theta \cos \theta L / \cos \theta, \\
D_{x}=-V\left(C_{n} \cos ^{2} \theta+C_{t} \sin ^{2} \theta\right) L / \cos \theta, \\
T_{x}=-\left(\Omega R^{2}\right)\left(C_{n} \cos ^{2} \theta+C_{t} \sin ^{2} \theta\right) L / \cos \theta .
\end{array}
$$

Las expresiones de los coeficientes de arrastre varían según la bibliografía empleada. Las formas más extendidas están basadas en la teoría del cuerpo esbelto, asumiendo que el efecto de cada pequeño filamento es significativo solo de forma local. De acuerdo con Gray y Hancock, éstos vienen dados por [6]:

$$
\begin{aligned}
C_{t} & =\frac{2 \pi \mu}{\ln \left(\frac{2 \lambda}{a}\right)-\frac{1}{2}}, \\
C_{n} & =\frac{4 \pi \mu}{\ln \left(\frac{2 \lambda}{a}\right)+\frac{1}{2}},
\end{aligned}
$$

siendo $\mu$ la viscosidad dinámica del fluido antes mencionada. Nótese que estos coeficientes no solo dependen de la geometría del flagelo, sino también de las propiedades del medio en el que se encuentre.

\section{ROBOT NADADOR CON ACTUACIÓN MAGNÉTICA EXTERNA}

Esta sección describe el desarrollo de robot nadador actuado exteriormente mediante el campo magnético inducido por la rotación de un imán permanente, colocado en el extremo de un brazo robótico. Antes de la descripción del prototipo se introducen brevemente algunas cuestiones relativas a este tipo de actuación. La información completa de este prototipo puede encontrarse en [19].

Como se ha indicado, este prototipo está basado en el presentado en [4]. La rotación del imán provoca un par en el robot, que produce a su vez la rotación de la hélice, permitiendo así el movimiento en la dirección del eje en ambos sentidos. El par $T$ generado por el imán viene dado por la expresión:

$$
T=m \times B,
$$

siendo $m$ la distancia perpendicular desde el imán al robot y $B$ el campo magnético aplicado. Para mayor información acerca de este tipo de actuación también se puede consultar [17].

\subsection{Descripción del prototipo}

La Figura 3 muestra las fotografías del prototipo de robot tipo flagelo bacteriano y del brazo robótico para su actuación. Como se observa en la Figura 3(a), el robot está formado por una pieza tipo cápsula de PLA (ácido poliláctico) de unos 2 $\mathrm{cm}$, diseñada en SolidWorks y fabricada con una impresora 3D, que aloja dos imanes de botón, y un flagelo helicoidal (fijo) de acero. Estos imanes son los responsables de la rotación de la cápsula y, por tanto, del desplazamiento del robot. Las características geométricas de la hélice son las siguientes: $a=0,7 \mathrm{~mm}, 2 R=7,6 \mathrm{~mm}, \lambda=4,45 \mathrm{~mm}$ y $\kappa=8,5$ vueltas. Con respecto al brazo robótico (Figura 3(b)), la propulsión del robot se lleva a cabo mediante la rotación del imán gracias a un motor de corriente continua Maxon A-max 32, con reducción GP 32 A $33: 1$, que es controlado a través de LabView mediante la controladora EPOS2 24/2 del fabricante (va conectada mediante cable microUSB al ordenador de control). El imán utilizado es de neodimio $(\mathrm{NdFeB})$ de grado N35 de polaridad axial y tiene forma cilíndrica, de diámetro de $20 \mathrm{~mm}$, y con un orificio central de 
$6 \mathrm{~mm}$, que coincide con el eje del motor. El imán está colocado de manera transversal al eje del motor, alojado en una estructura imprimida con una impresora 3D que permite centrar el efecto del campo magnético dejando la mitad del volumen libre y la otra mitad ocupada para el imán. De este modo, los polos del imán van a girar y, consecuentemente, la orientación del campo magnético variará, que hará que la rotación del imán provoque la rotación del robot. Para posicionar el motor con el imán se emplea el robot Mitshubishi PA-10, un brazo robótico con 7 grados de libertad. El motor Maxon está acoplado al mismo mediante un soporte también impreso en 3D.

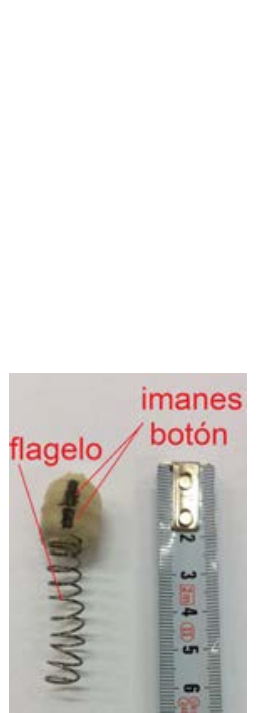

(a)

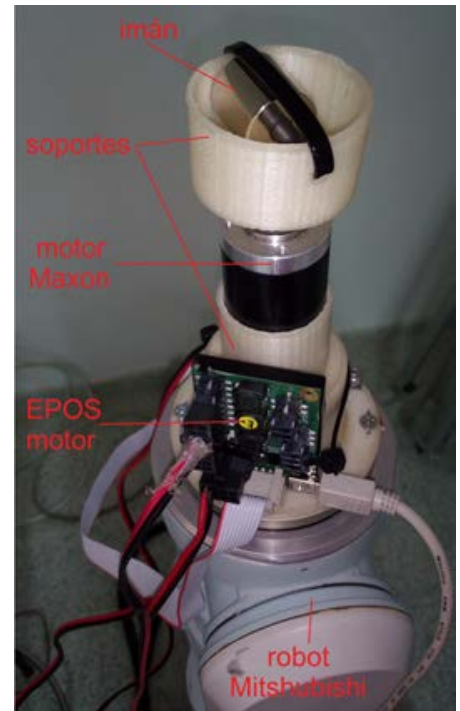

(b)
Figura 3: Prototipo de robot nadador con actuación magnética externa: (a) Robot (b) Brazo robótico para la actuación

\section{ROBOT NADADOR AUTOPROPULSADO}

Esta sección describe el desarrollo del robot nadador autopropulsado mediante un motor de pequeñas dimensiones, prototipo basado en el propuesto en [21]. La información completa del prototipo construido puede encontrarse en [22].

\subsection{Descripción del prototipo}

En la Figura 4 se muestra la fotografía del prototipo de robot tipo flagelo bacteriano autopropulsado. Como se observa, está constituido por una cápsula de PLA de unos $6,5 \mathrm{~cm}$, también diseñada en SolidWorks y fabricada con una impresora 3D, en cuyo interior alberga un motor de pequeñas dimensiones, modelo Pololu Micro Metal Gearmotor High Power 2218 y con reductora 298 : 1. Tanto la alimentación como el control de velocidad del motor se hace mediante un microcontrolador $\mathrm{Ar}$ duino $Y U ́ N$. El flagelo helicoidal es de acero tipo 304 y va acoplado al eje del motor mediante una pieza diseñada para tal fin. En este caso, las características geométricas del flagelo son: $a=0,7$ $\mathrm{mm}, 2 R=15,35 \mathrm{~mm}, \lambda=3,5 \mathrm{~mm} \mathrm{y} \kappa=17,5$ vueltas. No obstante, se disponen de otros flagelos con diferente geometría para probar. En este prototipo se considera solo el movimiento de la hélice; la cabeza está fija. Se están investigando técnicas de sellado adecuadas para la unión de la hélice al cuerpo del robot.

Cabe destacar que se ha desarrollado un modelo de simulación del prototipo en la herramienta Simscape de Simulink. Un vídeo de cómo se mueve el robot puede encontrarse en [23].

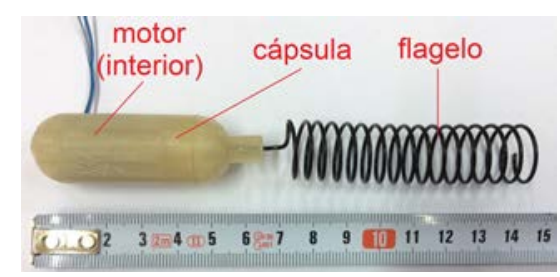

Figura 4: Prototipo de robot nadador autopropulsado

\section{EXPERIMENTOS}

Esta sección está dedicada a la descripción de las plataformas de prueba y de los experimentos realizados con cada uno de los prototipos desarrollados.

Dado que para ambos prototipos se pretende conseguir que el robot nade en condiciones de bajo $R e$, es necesario hacer unos cálculos previos antes de elegir el fluido en el que se llevarán a cabo los experimentos. Como se comentó en la sección 2, para que $R e$ en la expresión $(3)$ sea bajo $(<1)$ se debe utilizar un fluido que, a las velocidades de desplazamiento esperadas y una vez establecido el tamaño del robot, tenga una viscosidad cinemática, $\eta=\mu / \rho$, adecuada. En el caso que nos ocupa, suponiendo velocidades del orden de $\mathrm{cm} / \mathrm{s}$ y dadas las dimensiones de los robots (del orden de centrímetros), para cumplir la hipótesis de partida es necesario que el fluido venga caracterizado por $\eta<10^{-4} \mathrm{~m}^{2} / \mathrm{s}$. Se ha elegido un aceite de silicona PMX-200 del fabicante Xiameter de $\eta=100 \mathrm{cSt}$ (centistokes), es decir, $\eta=10^{-4} \mathrm{~m}^{2} / \mathrm{s}$ (consúltense sus características en [32]). 


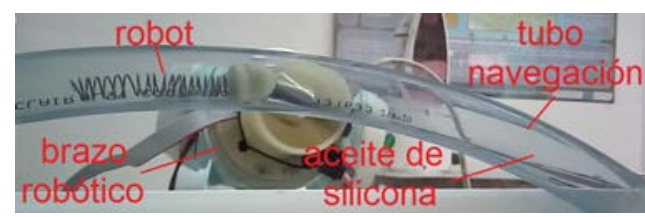

Figura 5: Plataforma experimental para el robot nadador con actuación externa

\subsection{Robot nadador con actuación externa}

La Figura 5 muestra la plataforma de prueba para el robot nadador con actuación magnética externa. Como puede verse, el banco de prueba consiste básicamente en un tubo de silicona rígido que se ha llenado con el aceite de silicona. Se introduce el robot en el tubo y se coloca delante del brazo robótico. El experimento se inicia llevando el brazo robótico a una posición cercana al robot nadador. A continuación se hace girar el imán permanente (mediante el motor) a una velocidad determinada. Esto induce el giro del robot, con velocidad proporcional a la del motor, y la correspondiente navegación a través del tubo, imitando el nado de una bacteria tipo flagelo. La Figura 6 muestra la relación entre la velocidad del motor y la del robot para diferentes distancias de separación entre el manipulador y el robot. El vídeo de uno de los experimentos realizados puede verse en [20].

Cabe mencionar un par de cuestiones referidas a los experimentos. Primero, queda pendiente la medida del campo magnético inducido. Segundo, se hicieron pruebas con el imán colocado en la misma dirección del eje del motor sin obtener los resultados esperados. En este caso, se intentó hacer nadar al robot oponiendo los campos magnéticos de los imanes. El resultado observado fue el arrastre del robot, manteniendo la distancia con el imán, pero sin nadar. Asimismo, se probó la navegación del robot en el tubo vacío. En este otro caso, por causa del rozamiento se observó que el robot únicamente se desplazaba si era arrastrado por el campo magnético.

\subsection{Robot nadador autopropulsado}

La plataforma de prueba desarrollada para el robot nadador autopropulsado se ilustra en la Figura 7. Como puede observarse consiste en una estructura metálica base, concebida principalmente para albergar los sensores y actuadores que conforman la bancada, que soporta un raíl sobre el que se desplaza un carrito que sujeta una pieza de aluminio en forma de "L" a modo de eje. Nótese que esta pieza termina dentro de un tanque de dimensiones $50 \times 20 \times 30 \mathrm{~cm}$ y sujetará al robot, una

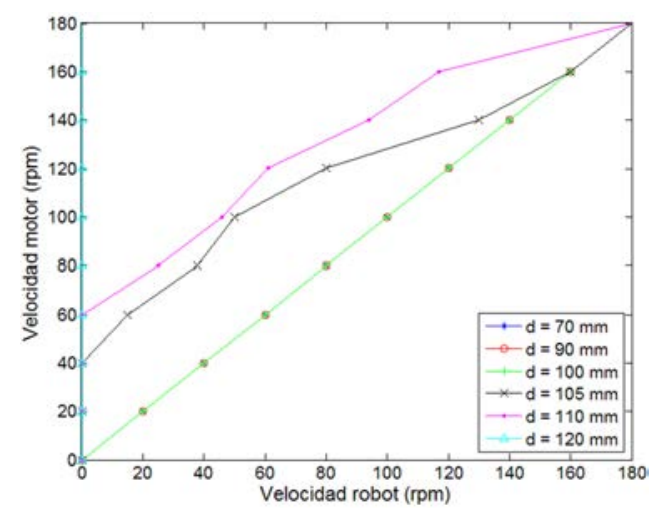

Figura 6: Relación entre velocidades para diferentes distancias $(d)$ de separación entre el actuador externo y el robot

vez sumergido, o la hélice en los experimentos. El tanque tiene una capacidad de 30 l y está lleno de aceite de silicona. En la parte superior del carro van unos agarres del eje en "L" con sus correspondientes rodamientos para que ésta pueda rotar libremente y unas piezas que sostienen una célula de carga, modelo Omega $L C L 227 G$, que permita medir las fuerzas de propulsión y arrastre del robot en los experimentos. En particular, la fuerza que se genere en el tanque hará que este eje rote y pince a la célula, provocando su deformación. El desplazamiento del carrito se hace mediante una cadena metálica que va acoplada a un engranaje insertado en un eje que mueve un motor Pololu 2288 en uno de los extremos del raíl. Mencionar que, aunque la idea original es poder llevar a cabo experimentos que permitan medir la fuerza de propulsión y la fuerza de arrastre del robot, se ha construido una estructura lo suficientemente grande como para ir haciendo mejoras y así incorporar también, por ejemplo, un experimento mediante el cual se pueda medir el par que ejerce la hélice al rotar.

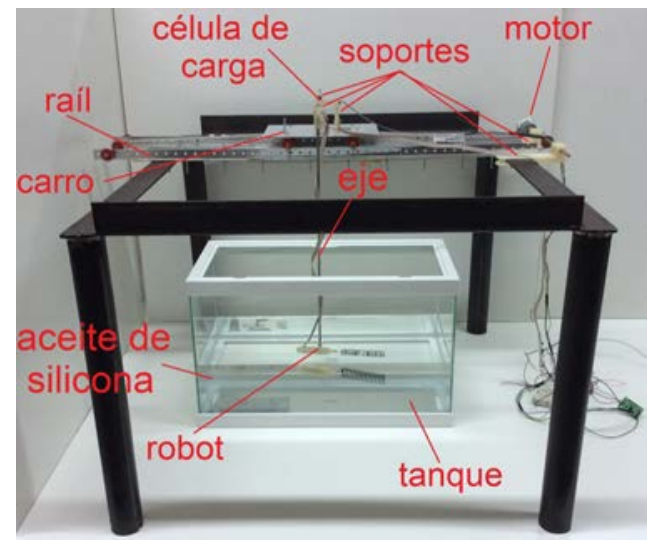

Figura 7: Plataforma experimental para el robot nadador autopropulsado 
Son dos los experimentos llevados a cabo sobre este prototipo: el primero, para medir la fuerza de propulsión del robot, mientras que en el segundo se mide la de arrastre. Es posible medir estas fuerzas con precisión una vez calibrada la célula. Esta calibración se ha realizado con un dinamómetro. De este prototipo queda pendiente la medida de estas fuerzas.

\subsubsection{Experimento 1: Medida de la fuerza de empuje}

En el primer experimento, el carrito permanecerá quieto, ya que se pretende medir la fuerza que ejerce el robot al hacer rotar la hélice, es decir, la fuerza que ejerce para autoimpulsarse. Por ello, se sumerge el robot en el tanque y se ancla en la punta del eje en "L" antes descrito. (Esto limita los movimientos que puede realizar el robot, y solo lo hará en una dirección). Se procede a mover el robot, actuando sobre su motor mediante un control de la velocidad de giro, implementado en el Arduino. Como ya se ha explicado, a medida que el robot se mueve, el eje rota y se deforma la célula de carga, cuya medida será la fuerza de propulsión del robot (transmitida a través del eje en "L"). La Figura 8 ilustra las fuerzas de empuje medidas con la célula de carga para diferentes tensiones de alimentación del motor. Un vídeo de uno de los experimentos realizados puede verse en [24].

Comentar que en este experimento se suponen pequeñas desviaciones del eje en "L", considerando, así, pequeño el error debido a que el robot debe empujar al eje y, a medida que empuja, más le cuesta seguir avanzando debido a que el ángulo que se forma entre la posición original del eje y la final es cada vez mayor. Consecuentemente, la fuerza del peso asociada al robot se desplaza de la vertical del eje.

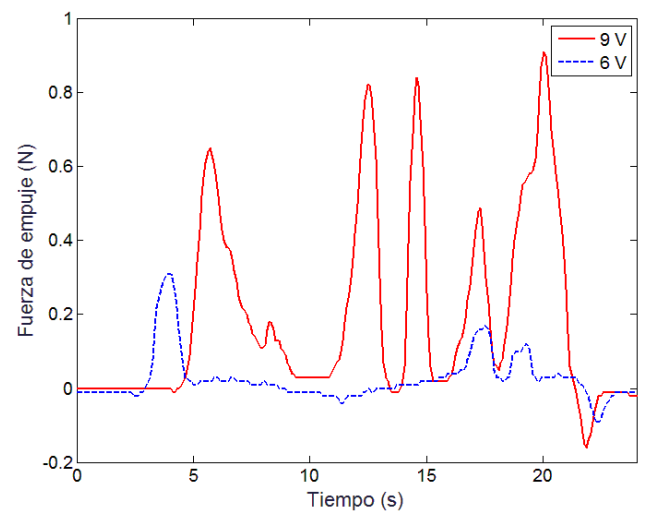

Figura 8: Resultados del experimento 1: fuerzas de empuje para diferentes voltajes de alimentación del motor

\subsubsection{Experimento 2: Medida de la fuerza de arrastre}

En el segundo experimento, se trata de medir la fuerza de arrastre que ejerce la hélice cuando ésta avanza en una dirección pero no rota. En este experimento se prescinde del cuerpo del robot. Para ello se ajusta la parte frontal del eje de la hélice a un acople de forma perpendicular al eje en "L" en el extremo sumergido. En este experimento se prescinde del cuerpo del robot. Debido al acople, la hélice también ve limitados todos sus movimientos, excepto en la dirección en la que se desplaza el carrito. En este caso, el movimiento de la hélice no será tan inmediato, ya que para moverse tendrá que superar el rozamiento del fluido. La fuerza de resistencia que se opone al movimiento en esa dirección de la hélice es lo que se conoce como fuerza de arrastre, la cual se medirá con la célula de carga mediante la deformación que sufra transmitida por el eje en "L". La Figura 9 muestra el resultado de uno de los experimentos realizados; el vídeo del mismo se puede ver en [25].

De nuevo, en este experimento vuelve a haber un pequeño error en este caso debido a que el arrastre obtenido en la medición no es solo el del flagelo helicoidal, sino que también se mide el arrastre producido por la parte sumergida del eje en "L". Para reducir este error lo máximo posible, se han tomado medidas de la fuerza de arrastre del eje sin la hélice a diferentes velocidades que se restarán a las obtenidas.

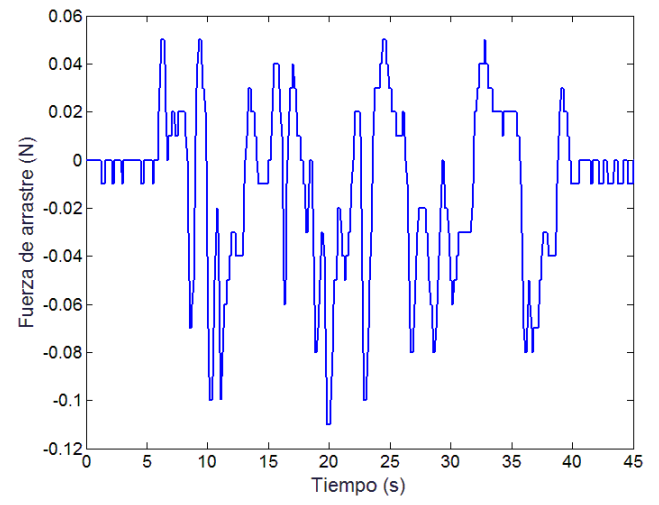

Figura 9: Resultados del experimento 2: fuerza de arrastre

\section{CONCLUSIONES}

Este artículo ha presentado las primeras experiencias de nuestro equipo de investigación en el desarrollo de robots nadadores tipo flagelo helicoidal de pequeñas dimensiones (de unos pocos centímetros) capaces de navegar en un fluido en condiciones de bajo número de Reynolds. El objetivo final 
de la línea de investigación iniciada con este trabajo es el desarrollo de microrrobots nadadores tipo flagelo artificial bacteriano capaces de navegar por el sistema circulatorio humano. Por tanto, los prototipos aquí presentados, debidamente escalados a dimensiones micro o nano con la tecnología de fabricación adecuada, pretenden ser los primeros pasos hacia nuevas soluciones técnicas para procedimientos médicos y quirúrgicos menos invasivos.

Sin embargo, a los desafíos ya impuestos por trabajar en la escala micro habría que sumarles los derivados de la navegación en la sangre. De modo que para diseñar, construir, controlar y aplicar microrrobots en medicina, y en particular en el sistema circulatorio humano, deben considerarse varios retos: cambios en las magnitudes relativas de las fuerzas que configuran la dinámica de un objeto móvil, requerimientos de energía que debido al tamaño no se pueden resolver utilizando los de la macroescala, procesos de fabricación innovadores para un robot pero muchos ya utilizados en microelectrónica y sistemas microelectromecánicos, y métodos de locomoción y control con características especiales debido a la interrelación entre el tamaño del robot y las características del entorno. Estos son solo los primeros pasos en esta dirección.

\section{Agradecimientos}

Este trabajo ha sido parcialmente financiado por la Ayuda a Grupos de la Junta de Extremadura con número de expediente GR15178.

\section{Referencias}

[1] Jake J. Abbott, Kathrin E. Peyer, Marco Cosentino Lagomarsino, Li Zhang, Lixin X. Dong, and Bradley J. Nelson. How should microrobots swim? The International Journal of Robotics Research, 28(11-12):1434-1447, 2009.

[2] R.R. Alfano, S. Alfano, Q.Z. Wang, and P.P. Ho. Remote-controllable, micro-scale device for use in in vivo medical diagnosis and/or treatment, May 29 2001. US Patent $6,240,312$.

[3] Nikolaus Correl and Alcherio Martinoli. Multirobot inspection of industrial machinery. IEEE Robotics \& Automation Magazine, pages 103-112, 2009.

[4] Thomas W. R. Fountain, Prem V. Kailat, and Jake J. Abbott. Wireless control of magnetic helical microrobots using a rotatingpermanent-magnet manipulator. In Proceedings of the IEEE International Conference on Robotics and Automation (ICRA 2010), pages 576-581, 2010.

[5] James Friend. Microrobot to swim like bacteria with flagellar propeller, 2006. Online: http://www.technovelgy.com/ct/ Science-Fiction-News . asp?NewsNum=845.

[6] J. Gray and G. J. Hancock. The propulsion of sea-urchin spermatozoa. Journal of Experimental Biology, 32:802-814, 1955.

[7] Christopher Brian House. Sonic Actuation of Small-scale Robots in a Fluidic Environment. Trident Scholar project report. 2014.

[8] Ian W. Hunter, Tilemachos D. Doukoglou, Serge R. Lafontaine, Paul G. Charette, Lynette A. Jones, Mark A. Sagar, Gordon D. Mallinson, and Peter J. Hunter. A teleoperated microsurgical robot and associated virtual environment for eye surgery. Presence, 2(4):265-280, 1994.

[9] G. Kosa, M. Shoham, and M. Zaaroor. Propulsion method for swimming microrobots. IEEE Transactions on Robotics, 23(1):137150, 2007.

[10] Vijay Kumar, Daniela Rus, and Sanjiv Singh. Robot and sensor networks for first responders. IEEE Pervasive Computing, 3(4):24-33, 2004.

[11] H. Li, J. Tan, and M. Zhang. Dynamics modeling and analysis of a swimming microrobot for controlled drug delivery. IEEE Transactions on Automation Science and Engineering, 6(2):220-227, 2009.

[12] Nikhil Londhe, R. Majumdar, J.S. Rathore, and N.N. Sharma. Simulation of planar wave flagellar propulsion of nanorobots using COMSOL. In Excerpt from the Proceedings of the 2011 COMSOL Conference, pages 1-3, 2011.

[13] Veronika Magdanz, Samuel Sanchez, and Oliver G. Schmidt. Development of a spermflagella driven micro-bio-robot. Advanced Materials, 25:6581-6588, 2013.

[14] R. R. Murphy, J. Kravitz, S. L. Stover, and R. Shoureshi. Mobile robots in mine rescue and recovery. IEEE Robotics Automation Magazine, 16(2):91-103, 2009.

[15] Bradley J. Nelson, Ioannis K. Kaliakatsos, and Jonh J. Abbott. Microrobots for minimally invasive medicine. Annual Review of Biomedical Engineering, 12(1):55-85, 2010. 
[16] Igor Paprotny and Sarah Bergbreiter. SmallScale Robotics. From Nano-to-MillimeterSized Robotic Systems and Applications, volume 8336 of Lecture Notes in Computer Science, chapter Small-Scale Robotics: An Introduction, pages 1-15. Springer-Verlag Berlin Heidelberg, 2014.

[17] Kathrin E. Peyer, Arthur W. Mahoney, Li Zhang, Jake J. Abbott, and Bradley J. Nelson. Microbiorobotics. Biologically Inspired Microscale Robotic Systems, chapter Bacteria-Inspired Microrobots, pages 165199. Elsevier, 2012.

[18] Kathrin E. Peyer, Li Zhang, and Bradley J. Nelson. Bio-inspired magnetic swimming microrobots for biomedical applications. Nanoscale, 5:1259-1272, 2013.

[19] Javier Prieto Arranz. Equipo electromagnético para propulsión y guiado de microrrobots nadadores tipo flagelo bacteriano helicoidal. Trabajo Fin de Grado, Escuela de Ingenierías Industriales, Universidad de Extremadura, Septiembre 2016.

[20] Javier Prieto Arranz. Robot nadador tipo flagelo bacteriano de pequeñas dimensiones con actuación magnética externa: experimento (vídeo), 2016. Online: http://158.49.55.50/owncloud/index. $\mathrm{php} / \mathrm{s} / \mathrm{P} 3 \mathrm{SSKIn} 31 \mathrm{Ve} 9 \mathrm{~A} 8 \mathrm{~T}$.

[21] Bruce Rodenborn, Chih-Hung Chen, Harry L. Swinney, Bin Liu, and H. P. Zhang. Propulsion of microorganisms by a helical flagellum. Proceedings of the National Academy of Sciences of United States of America, 110(5):E338-E347, 2013.

[22] Javier Silva García. Diseño de un robot tipo flagelo artificial helicoidal autopropulsado y de la plataforma de pruebas asociada. Trabajo Fin de Grado, Escuela de Ingenierías Industriales, Universidad de Extremadura, Julio 2016.

[23] Javier Silva García. Robot nadador tipo flagelo bacteriano autopropulsado de pequeñas dimensiones: simulación en Simscape (vídeo), 2016. Online: http://158.49.55.50/ owncloud/index.php/s/T5gDTT7CthL19qt.

[24] Javier Silva García. Robot nadador tipo flagelo bacteriano autopropulsado de pequeñas dimensiones: experimento 1 (vídeo), 2016. Online: http://158.49.55.50/owncloud/ index. php/s/KJBtmNlXgSEx 4 WJ.
[25] Javier Silva García. Robot nadador tipo flagelo bacteriano autopropulsado de pequeñas dimensiones: experimento 2 (vídeo), 2016. Online: http://158.49.55.50/owncloud/ index.php/s/PLHvY7iyJ5zXeRs.

[26] Metin Sitti. Microscale and nanoscale robotics systems: Characteristics, state of the art, and grand challenges. IEEE Robotics \&6 Automation Magazine, 14(1):53-60, 2007.

[27] D. A. Stepanenko, V. T. Minchenya, R. M. Asimov, and K. Zimmermann. Possibility of application of small-size robots with vibratory piezoelectric actuators for inspection of physical state of surfaces. In Proceedings of the 2011 International Congress on Ultrasonics - Gdansk 2011, 2011.

[28] Seiichi Sudo. Biomimetics Learning from $\mathrm{Na}$ ture, chapter Micro Swimming Robots Based on Small Aquatic Creatures, pages 343-362. InTech, 2010.

[29] José Emilio Traver, Blas M. Vinagre, and Inés Tejado. Robot nadador tipo flagelo bacteriano plano: Estudio y simulación del mecanismo de propulsión. In XXXVII Jornadas de Automática, 2016.

[30] Manuela Veloso, Peter Stone adn Kwun Han, and Sorin Achim. Team Description SmallSize Robot Teams. RoboCup-97: Robot Soccer World Cup I, volume 1395 of Lecture Notes in Computer Science, chapter The CMUnited97 small robot team, pages 242-256. Springer Berlin Heidelberg, 1998.

[31] R. M. Voyles and A. C. Larson. TerminatorBot: a novel robot with dual-use mechanism for locomotion and manipulation. IEEE/ASME Transactions on Mechatronics, 10(1):17-25, 2005.

[32] Xiameter. Características del aceite de silicona PMX-200 de 100 cSt. Online: https : //www.xiameter.com/EN/Products/Pages/ ProductDetail . aspx?halb=4088892.

[33] L. Zhang, K. E. Peyer, T. Petit, B. E. Kratochvil, and B. J. Nelson. Motion control of artificial bacterial flagella. In Proceedings of the 10th IEEE Conference on Nanotechnology (IEEE-NANO 2010), pages 893-896, 2010.

[34] Raziq Asyraf Zin, Khairul Salleh Mohamed Sahari, Juniza Md Saad, Adzly Anuar, and Abd Talip Zulkarnain. Development of a low cost small sized in-pipe robot. Procedia Engineering, 41:1469-1475, 2012. 